\title{
LA ENSEÑANZA DE LAS CIENCIAS Y LA FORMACIÓN DE PROFESORES DE ENSENANZA PRIMARIA PARA LA REFORMA CURRICULAR: DE LA TEORÍA A LA PRÁCTICA
}

\author{
PAIXAO, M. DE FÁTIMA ${ }^{1}$ y CACHAPUZ, ANTONIO ${ }^{2}$ \\ ${ }^{1}$ Escola Superior de Educação. 6000 Castelo Branco. Portugal. \\ ${ }^{2}$ Departamento de Didáctica e Tecnologia Educativa. Universidad de Aveiro. 3800 Aveiro. Portugal.
}

\begin{abstract}
SUMMARY
This papers presents a qualitative study aiming to investigate how new Portuguese curriculum proposals from primary school (the intentional curriculum) are translated in the teaching of science of four prospective teachers (the operational curriculum).

The research adopted a case study methodology in order to describe main features of the pedagogical profile of classroom practices of those teachers. Three different profiles were identified. Some interpretative aspects are outlined and important implications for pre-service education are put forward.
\end{abstract}

\section{INTRODUCCIÓN}

El hombre llegó a la Luna pero, paralelamente a este progreso, ha ido creciendo el número de personas científicamente ignorantes. Se ha hecho bien poco para reducir la ignorancia científica de los niños al finalizar el ciclo elemental de sus estudios. El problema es importante (UNESCO, 1986) y justifica, en buena parte, la idea de que son necesarios algunos cambios en la orientación actual de la enseñanza de las ciencias. Según nuestra opinión, este cambio de perspectiva pasa por el reconocimiento de los más recientes avances en el campo de la didáctica constructivista. Estos avances, al valorar las aportaciones de la psicología del aprendizaje y de la epistemología, permiten dar sentido y coherencia a un nuevo diálogo entre los conocimientos del alumno y los de las propias disciplinas.

Ha sido necesario recorrer un largo camino hasta la aparición de esta nueva perspectiva. En un ambiente de aparente normalidad, la pedagogía, en los vastos proyec- tos de los años sesenta y setenta, encaró el aprendizaje como un proceso de carácter secuencial, es decir, que se desarrolla a partir de la observación y finaliza con una conclusión teórica, aplicando un método lineal operativo como forma de adquisición de los conceptos científicos. El lema era formar científicos a imagen del paradigma científico. De la evaluación de estos proyectos surgió la convicción de que era necesario algo diferente, o sea, de que se había de ofrecer cultura científica al ciudadano, alfabetizarlo científicamente. Por esto, la perspectiva de las reformas curriculares de los años ochenta ya no podía ser aquella inicial, sino que debía cambiar. A partir de entonces las reformas curriculares tienen como base una nueva imagen de la ciencia, en la cual se realza el carácter constructivo de los conocimientos (Fourez, 1988; Geymonat, s/d).

El vacío creado al no poder aplicar el esquema epistemológico positivista condujo a una reflexión sobre el cono- 
cimiento científico cuyo resultado caracteriza la situación actual. Se combina ahora la pluralidad metodológica con una mayor personalización del trabajo científico y se valora especialmente la calidad del conocimiento adquirido. En consecuencia, el cambio más importante realizado en este campo es que se ha priorizado que el saber científico pueda ser aprovechado por el ciudadano; o sea, que se ha proporcionado al conocimiento un nuevo sentido común (Santos, 1987). La vertiente epistemológica, entendida como discusión sobre la construcción del conocimiento científico, enlaza con la dimensión psicológica del aprendizaje de los alumnos, es decir, con la manera con que éstos se apropian del saber científico (Cleminson, 1990). Las perspectivas teóricas del aprendizaje se desvían así hacia el campo del cognitivismo, de manera que queda claro para diversos autores, en la enseñanza de las ciencias, que hoy en día se reconoce al alumno como autor de la construcción de su propio conocimiento (Taylor y Cheung, 1991). Esta construcción incluye características de la persona, tales como conocimientos anteriores, habilidades y aptitudes que tienen su origen en experiencias, en la herencia genética y también en el contexto en el que se desarrolla el aprendizaje (White, 1988). De todo esto surgen las propuestas de Ausubel incentivando un tipo de enseñanza que se basa en el conocimiento previo de los alumnos y no en la organización de secuencias previamente programadas y basadas exclusivamente en la estructura de la disciplina (Novak, 1977).

La importancia de formar ciudadanos científicamente «alfabetizados» implica una manera diferente de enfrentarse a la enseñanza de las ciencias. Pero esto no es suficiente. Según Harlem (1985), es necesario iniciar a los niños en el estudio de las ciencias lo antes posible, promoviendo la educación de sus procesos, conceptos y actitudes. Éstas son las dos líneas fundamentales que deberían enmarcar, tanto en el espacio como en el tiempo, las propuestas futuras de las reformas curriculares (Wallace y Louden, 1992).

\section{LAS REFORMAS CURRICULARES Y LA SITUACION DE LAS PRACTICAS PEDA- GOGICAS}

Mitter (1991) reconoce el enorme esfuerzo que fue necesario realizar en los sistemas escolares donde fueron concebidas e implementadas determinadas reformas. Sin embargo, la comparación con otros países demuestra, como indica el propio autor, que en muchos de ellos la implicación de los profesores se presenta como el anillo más débil de la cadena, tanto en los documentos teóricos como en las declaraciones políticas y en las reglamentaciones. Y esto es un problema, ya que a ellos corresponde adaptar las reformas a la realidad de cada centro educativo.

Abell y Roth (1992) alertan: «We need teachers who are enthusiastic toward teaching science», completando, de este modo, el «cuadro negro» de la educación de las ciencias a nivel elemental e indicando que la ciencia ha sido el tema académico más olvidado en las escuelas primarias de los Estados Unidos. La justificación que Mullis y Jenkins (1988, en Abell y Roth, 1992) presentan para este hecho es que los profesores de nivel elemental se sienten poco cualificados y poco seguros cuando enseñan ciencias.

Duschl (1983) ha tratado la cuestión de la difícil reconciliación que debe ser llevada a cabo por los futuros profesores de nivel elemental entre los cursos que enfatizan el conocimiento de los contenidos y los que se refieren a los métodos pedagógicos, principalmente orientados hacia la manera de proceder en clase. El autor añade que, para resolver este antagonismo, los futuros profesores manifiestan la tendencia a mantenerse fieles a la enseñanza de las ciencias como un bloque de conocimientos; es decir, consideran las ciencias como una colección de hechos y figuras que ellos mismos memorizan. Todas estas dificultades en la práctica de la enseñanza de las ciencias son apuntadas por Stefanich y Kelsey (1989) y también por Cronin-Jones y Shaw (1992), que avanzan la necesidad de realizar importantes cambios en la formación del profesorado.

En Portugal, el Ministerio de Educación desarrolló un conjunto de estudios curriculares (GEP, 1989) «con el objetivo de analizar y evaluar de manera sistemática los currículos de la enseñanza obligatoria, así como avanzar datos significativos para la reflexión y la planificación» (op. cit., p. 11). Se pretende dar respuesta a «dónde, quién y cómo se enseña en Portugal». En este análisis destaca que el tiempo medio dedicado a la enseñanza del medio físico y social (o sea, las ciencias impartidas conjuntamente con las sociales) es bastante inferior al que se dedica a la lengua portuguesa y a las matemáticas, aunque se sitúa por encima de las restantes disciplinas. En lo referente a las conclusiones para caracterizar la práctica pedagógica, se comprueba que la enseñanza se basa en la consecución de objetivos. Así pues, el programa oficial y los manuales escolares son fundamentales en el aula y ambos son competencia del profesor. Se ha constatado que el trabajo individual tiene más importancia que el trabajo colectivo y también que la lengua, las matemáticas, el estudio del medio físico y social, y las actividades que de estas disciplinas se desprenden son las que ocupan un mayor porcentaje de horas semanales en la enseñanza (op. cit., p. 96)

Jorge (1987) presentó un estudio minucioso en el cual se articula la cuestión: «¿Qué enseñanza? ¿Con qué formación?»E intenta analizar en qué medida la formación recibida por el profesor de ciencias se relaciona con su práctica pedagógica (op. cit., p. 6). El estudio se centra en el segundo ciclo de la enseñanza básica (10-12 años) y las conclusiones a las cuales llega son, en resumen, que la práctica docente de los profesores no siempre parece coherente con los objetivos que presidían su formación, particularmente respecto al dominio sociopsicopedagógico. Así, esta falta de coherencia parece estar íntimamente relacionada con deficiencias existentes en la formación inicial de profesores. También se ha observado que por parte de la universidad existen una serie de contradicciones entre una teoría innovadora y una prác- 
tica de carácter más conservador (op. cit., pp. XIII, XIV).

En un artículo dedicado a la enseñanza de las ciencias en el primer ciclo de la enseñanza básica en Portugal (6-9 años), Cachapuz (1992) hace referencia a las dificultades en el área de ciencias que experimentan los profesores de estos niveles y también al incentivo para desarrollar estrategias innovadoras en el área de ciencias. El autor equipara la propuesta de reforma curricular portuguesa a las iniciadas ya en otros países, principalmente europeos, en los cuales las mayores modificaciones que se están llevando a cabo son: la inclusión de nuevas áreas conceptuales, una perspectiva constructivista del aprendizaje y un mayor énfasis en el tratamiento experimental de la enseñanza de las ciencias, consistiendo este último en la necesidad que tiene el profesor de confiar en su conocimiento científico, de adoptar un papel facilitador del aprendizaje y de ser apto para ayudar a los niños a desarrollar y usar los procedimientos y actitudes científicos. Respecto a las estrategias de enseñanza y al conocimiento científico, los resultados obtenidos apuntan hacia la importancia de la función del profesor y también muestran que la experiencia profesional no se observa como una variable decisiva en los profesores de enseñanza de ciencias que presentan métodos más innovadores. Los resultados también sugieren que en la formación continuada es necesario poner mayor énfasis en la forma de explorar las ideas intuitivas de los niños sobre los conceptos científicos y cómo dirigirlos hacia la formulación de hipótesis, la experimentación, el análisis de datos y, finalmente, la confrontación de los resultados. En relación con la competencia científica de los profesores de enseñanza básica, en relación con los contenidos, Costa y otros (1993) avanzan unos resultados que sugieren que la enseñanza está muy lejos de ser la óptima, sobretodo en el área de física y química, no tanto en biología.

En un estudio del Instituto de Innovación Educacional (IIE, 1992) se apunta la más que probable dificultad de implementar en los años noventa la nueva reforma curricular portuguesa, dada la inadecuada preparación o situación profesional de los profesores, especialmente con relación a las ciencias. Entre otros aspectos, se cuestiona cómo se incorporarán los nuevos programas al aula y qué necesidades se podrán identificar respecto a la formación de profesores. Los más de dos mil profesores entrevistados, de nivel de enseñanza no superior, reconocen que la falta de formación y de recursos serán los condicionantes fundamentales para conseguir la implementación de los nuevos programas en el aula (op. cit., pp. 1-4).

El tema de la formación del profesorado aparece como un denominador común en los estudios realizados y emerge como la problemática central cuando lo que se quiere es catalizar y conducir un proceso innovador en la enseñanza de las ciencias. En particular, en lo referente a la formación inicial de profesores de primer ciclo de enseñanza básica (CEB), parece importante saber cómo interpretan y trasladan las recientes propuestas de innovación curricular en Portugal a sus prácticas pedagógicas.

\section{EL PROBLEMA A INVESTIGAR}

Nuestro punto de partida, basado en la investigación teórica realizada, es que, en la formación inicial de profesores, no va a ser implementada una práctica pedagógica (PP) de ciencias naturales congruente con los principios orientadores propuestos por la reforma curricular portuguesa para el primer ciclo de enseñanza básica.

El tipo de PP desarrollada por los profesores en período de formación (PF) estará basada en diversos aspectos como: su formación anterior, sus experiencias de formación durante el curso, sus propias convicciones sobre la enseñanza, el aprendizaje y las representaciones del profesor que se transmitan en la práctica pedagógica de final de curso.

Teniendo ya identificada la problemática y situado el campo de estudio -formación inicial de profesores-, definiremos los objectivos de la investigación:

1. Describir la práctica pedagógica $(\mathrm{PP})$ del área de ciencias de los profesores en formación (PF) de primer ciclo de enseñanza básica (CEB).

2. Analizar las discrepancias entre el cuadro preconizado por la reforma en curso (currículo intencional) y la práctica pedagógica (currículo operacional).

3. Proponer cambios en la formación inicial de profesores de primer ciclo de enseñanza básica que vehiculen mejoras en la enseñanza de las ciencias en las escuelas elementales portuguesas.

\section{METODOLOGÍA}

El estudio se ha realizado según una metodología cualitativa de estudio de casos (Yin, 1987), que ha permitido no tan sólo investigar la naturaleza de las prácticas pedagógicas de ciencias en los profesores en formación, sino también sus representaciones sobre tales prácticas. Los procedimientos para llevar a cabo el estudio han sido el análisis documental, el cuestionario, la observación de prácticas pedagógicas y la entrevista. De manera genérica, el modelo que se ha desarrollado en la investigación se presenta en la figura 1. Nuestra intención ha sido, a partir del análisis realizado, llegar a nuevas perspectivas, base de los cambios necesarios en la práctica pedagógica, haciendo a esta última, por supuesto, coherente con las tendencias internacionales de reforma curricular.

Seguidamente procederemos al análisis de los textos de la reforma curricular y en particular de los nuevos programas para el primer ciclo de enseñanza básica en el área de ciencias. Se intuyen cinco principios orientadores de cómo debe ser el aprendizaje: activo, significativo, diversificado y creativo, integrador y globalizador, y finalmente socializador. A partir de estas premisas cons- 
truimos el cuadro teórico de referencia que contiene la práctica pedagógica que presentan los efectos teóricamente esperados y que puede ser utilizado como instrumento de análisis (Cuadro I). Un proceso inferencial como éste fue validado independientemente por dos jueces especialistas en educación de ciencias.

Cuadro I

Categorías de aprendizaje y enseñanza.

\section{CATEGORÍAS DE APRENDIZAJE Y ENSEÑANZA}

Indicadores de análisis de prácticas pedagógicas

Aprendizaje (A): Activo.

Enseñanza (E): Articulación de contenidos con procesos.

Indicadores (I): Desarrollo de proyectos y recursos de investigación por parte de los alumnos.

\section{A: Significativo.}

E: Relación del dominio cognitivo con el afectivo.

I: Situaciones que exploren y fomenten la experiencia y vivencia cotidiana de los alumnos, y en la cual se proceda al diagnóstico y cambio de sus concepciones alternativas.

A: Diversificado y creativo.

E: Utilización de recursos didácticos variados y de formas de trabajo diversas y creativas.

I: Diversificación de metodologías de enseñanza y uso de materiales accesibles (por ejemplo, modelos), eventualmente promoviendo su construcción por parte de los alumnos.

\section{A: Integrador y global.}

E: Interrelación de diferentes áreas de los contenidos y de éstos con los procesos y actitudes.

I: Influencia de otras áreas (lengua materna, expresiones...), aplicación de procesos y actitudes de las ciencias en la resolución de problemas de otras áreas.

\section{A: Socializador.}

E: Aspectos de las integraciones en pequeños grupos y en el grupo-clase.

I: Situaciones en las cuales los niños se mueven y hablan informalmente, manteniendo en nivel de participación y de interés elevados.

\section{Selección de la muestra}

Fueron estudiados cuatro profesores en formación del último año del curso de formación de profesores de CEB, que trabajaron con un profesor cooperante (orientador). Estos casos son designados de A hasta D. La selección se realizó sobre la base de los siguientes criterios: disponibilidad para participar en el estudio, diferente trayectoria académica anterior al curso, diferente profesor cooperante y diferente institución formadora de profesores. El caso A presentaba un recorrido académico más extenso que el resto y con el mismo profesor cooperante que en el caso B; C tenía un profesor cooperante diferente de los casos anteriores y D pertenecía a una institución formadora de profesores diferente a los otros casos.

\section{Observación de la práctica pedagógica}

El objetivo de la observación en el aula fue recoger evidencias de los indicadores predefinidos y representativos de los comportamientos teóricamente previstos, para compararlos con el cuadro, llegando así, eventualmente, al establecimiento de perfiles diferentes. Fueron grabadas en vídeo un total de 23 horas de enseñanza y a partir de aquí se fueron elaborando determinados protocolos escritos (Paixao, 1993), previamente validados por los respectivos autores como descriptivos de su práctica pedagógica. Estos elementos constituyen la unidad básica del análisis.

\section{Entrevistas}

Después del análisis de las respectivas clases, procedimos a realizar entrevistas individuales con los profesores en formación (con una hora y media por cada profesor) para clarificar algunos aspectos de la observación, de las representaciones del aprendizaje de los alumnos y de la naturaleza de la ciencia. Todo esto también nos permitirá avanzar algunas interpretaciones, dadas las incongruencias detectadas entre el cuadro modelo y las prácticas pedagógicas desarrolladas. En relación con las entrevistas, fueron elaborados protocolos escritos (Paixao, 1993).

\section{RESULTADOS Y ANÁLISIS}

De acuerdo con Yin (1987), «lo más importante es tener una estrategia general de análisis» y «tener también alternativas interpretativas». Nuestra estrategia de análisis fue relacionar sistemáticamente las propuestas teóricas con la práctica, intentando dar respuesta al «cómo» y al «por qué», o sea, hacerla interpretativa y descriptiva a la vez. A partir del análisis global de la interpretación de la práctica pedagógica en relación con cada una de las categorías y con cada uno de los cuatro profesores, se establecieron y caracterizaron tres perfiles de prácticas pedagógicas en los cuatro profesores en período de formación implicados en el estudio (Cuadro II).

\section{Perfiles establecidos y factores interpretativos}

El caso A presenta un perfil de práctica pedagógica que se sitúa en el nivel de congruencia del cuadro teórico de referencia, superior a los otros casos analizados, pero sin llegar al grado deseado. En relación con la enseñanza del aprendizaje activo, no hemos podido encontrar en ninguna práctica pedagógica de los profesores estudiados la utilización de proyectos de investigación como estrategia para el aprendizaje. En determinados aspectos, el 
Perfiles de práctica pedagógica de los profesores en formación.

\begin{tabular}{|c|c|}
\hline $\begin{array}{l}\text { Profesor } \\
\text { en formación }\end{array}$ & PERFIL DE PRÁCTICA PEDAGÓGICA \\
\hline $\mathbf{A}$ & $\begin{array}{l}\text { Ciencia interpretativa: Importancia de la explicación de conceptos; abundantes preguntas; participación de los alumnos; } \\
\text { interés por las ideas de los alumnos; recursos audiovisuales abundantes y bien integrados; inclusión y referencia a aspectos } \\
\text { de la ciencia; integración de la ciencia y de otras áreas; transferencia para el ámbito tecnología, sociedad y cuestiones } \\
\text { ambientales; mucho entusiasmo; agradable ambiente en clase; interés por parte de los alumnos; papel incentivador y } \\
\text { dinamizador del profesor. }\end{array}$ \\
\hline B & $\begin{array}{l}\text { Ciencia ilustrada: Importancia de los ejemplos e ilustraciones, valorización del aspecto lúdico y de sorpresa hacia los } \\
\text { materiales; demostraciones en las cuales los alumnos algunas veces ayudan; escasas preguntas; mucha importancia } \\
\text { otorgada a los términos; sugestiones de los alumnos sin apenas ejemplos; recursos diversificados e ilustrativos; alumnos } \\
\text { pasivos y espectadores; falta de integración de conceptos de ciencias; relación entre diferentes áreas; implicaciones poco } \\
\text { discutidas de los conceptos del ámbito tecnológico, social y ambiental; nivel elevado de interés de los alumnos; papel del } \\
\text { profesor como conductor del aula; buena relación afectiva. }\end{array}$ \\
\hline $\mathrm{C}$ & $\begin{array}{l}\text { Ciencia descriptiva: No existe trabajo experimental de ninguna naturaleza; escasas cuestiones; poco interés por las } \\
\text { cuestiones de los alumnos; en ninguna ocasión se parte de situaciones cotidianas; estrategia de fortalecimiento de } \\
\text { información lineal y secuencial, esencialmente con exposición oral; pocos medios audiovisuales; presentación de secuencias } \\
\text { de definiciones que serán copiadas en el cuaderno; refuerzo mediante fichas; preguntas que inciden en las definiciones del } \\
\text { profesor; poca transferencia de conceptos para el ámbito de tecnología, sociedad y cuestiones ambientales; se evita que los } \\
\text { alumnos hablen; alumnos con reducido interés; discordias frecuentes. }\end{array}$ \\
\hline $\mathrm{D}$ & $\begin{array}{l}\text { Ciencia ilustrada: Abundantes demostraciones usadas como ilustraciones en el aula; ayuda de los mismos compañeros; el } \\
\text { profesor aprovecha el efecto de impacto y sorpresa de los materiales; poco espacio para el planteamiento de preguntas; } \\
\text { llamada al saber factual y a la memorización; fichas de evaluación como refuerzo; parte de ejemplos del conocimiento } \\
\text { cotidiano de los alumnos; frecuentemente retira la palabra al alumno; intervenciones desorganizadas; aprovecha únicamente } \\
\text { las intervenciones que son secuencia de su programación; recursos variados y abundantes; alumnos interesados; profesor- } \\
\text { conductor del aula; relación que oscila entre el buen humor y la agresividad. }\end{array}$ \\
\hline
\end{tabular}

caso A destaca del resto, ya que coloca a los alumnos en constante actividad física y sobretodo crea actitudes de reflexión; por ejemplo, cuando se estudian los movimientos relativos al Sol y la Tierra: «Puedes explicar [...] ahora con una bombilla [...] lo que ocurre en este movimiento, con la luz de la bombilla [...] Pensad y después decid [...]» Respecto a los profesores, son prácticamente nulas las evidencias para identificar enseñanza con aprendizaje activo.

Respecto a la enseñanza del aprendizaje significativo, sobresale entre todos el caso A, que valora las ideas de los alumnos: «¿Alguien sabe lo que es la gravedad? [...] Creo que sí que lo saben.» Da valor a la influencia de las concepciones alternativas de los alumnos respecto al aprendizaje de los conceptos. El resto de casos, en cambio, parece no tener en cuenta esta influencia y tampoco ofrece a los alumnos en referencia al oportunidades para hablar, excepto en respuestas de tipo confirmatorio: «¿Pensáis que el aire tiene fuerza o que no tiene?»(Caso B). Es notorio el énfasis que se da a simples aspectos ilustrativos. Así, por ejemplo, en el estudio de la energía solar, en el caso B, se comenta: «Hoy os he traído una sorpresa (panel solar)», sin intentar investigar desde el inicio la problemática de los alumnos y no dando valor a las ideas que éstos puedan tener respecto al fenómeno. A pesar de esos aspectos meramente ilustrativos, extensivos también al caso D, existe un interés manifiesto de estos profesores en perío- do de formación por el área de ciencias: «Me gustó, me gustó mucho [...] es un tema que me fascina.» (entrevista B). «Me gusta. Creo que éstas son el tipo de clases en las cuales los alumnos están más interesados.» (entrevista D).

En cuanto al rigor científico, el lenguaje utilizado por A es el más adecuado, posiblemente por ser el más utilizado académicamente en el área de ciencias. Encontramos incorrecciones científicas en todos los casos estudiados y algunos incluso revelan muchas dificultades en el dominio de los conceptos científicamente aceptados.

Con relación a la representación del aprendizaje de las ciencias, todos los casos se caracterizan por dar excesiva importancia a la observación, menospreciando la importancia de las preguntas, de la formulación de hipótesis y las tentativas para resolver los problemas, tal como defiende Gil Pérez (1983) y también Carrascosa y Gil Pérez conjuntamente (1985). Respecto a las representaciones sobre el trabajo científico, A es el caso al cual atribuimos actitudes menos dogmáticas de comprensión de la ciencia y del trabajo científico. El caso $\mathrm{C}$ es el que más se aleja, de hecho, del cuadro teórico y reconoce que «también las ciencias estuvieron lejos de mi interés.» (entrevista $\mathrm{C})$.

Respecto a la enseñanza como un aprendizaje integrador encontramos algunos logros en lo se refiere a la articu- 
lación de las actividades y áreas. Las prácticas A y D se centran en aspectos como la integración de las ciencias, la sistematización de los conceptos, la transferencia del aprendizaje en situaciones cotidianas y la discusión de conceptos. El caso A parece relacionarse con su interés personal, dada su trayectoria académica en el área de ciencias. El caso D, en su entrevista, atribuye alguna influencia al profesor cooperante, ya que le ha hecho pensar en aspectos relacionados con la ciencia que anteriormente le pasaban por alto (por ejemplo, la utilización de materiales didácticos y también la relación entre conceptos científicos y su tratamiento cotidiano). El caso B presenta mucha dificultad para realizar la integración de ideas comunes, avanzadas ya por los mismos alumnos en determinados períodos de enseñanza, aunque hace algunas tentativas. El caso $C$ se muestra incapaz de cualquier transferencia y explotación de conceptos.

El aspecto socializador difiere en los cuatro casos. El nivel de interés de los alumnos es muy diversificado, dependiendo de la actividad del profesor en prácticas; en general, los alumnos participan con entusiasmo en los diálogos y en las actividades de grupo. Cuando los profesores pretenden ofrecer información, surge confusión y desorden, principalmente en el caso C. Parece como si no existieran reglas de trabajo definidas en ninguno de los casos, aunque todos afirmen que sí que las establecieron. El caso D tiene en sus clases períodos de intenso desorden, intercalados con otros en los cuales los alumnos manifiestan interés por las actividades realizadas. El intercambio de información para el conjunto de la clase respecto a las conversaciones orientadas prácticamente no existe. A permite que los alumnos interroguen espontáneamente. En general, el trabajo de grupo parece surgir de la necesidad de aparentar una «enseñanza moderna». D es quien menos recurre al trabajo de grupo.

Con relación a los atributos que definen los perfiles que hemos identificado y que caracterizan la práctica pedagógica (PP) de cada uno de los profesores (Cuadro II), la designación de un perfil de enseñanza de ciencia interpretativa (caso A) coincide con la preferencia por las ciencias que coincide con una mayor trayectoria académica en ciencias -ya que el profesor estudió de forma continuada física, química y biología hasta el final de la escuela secundaria- y, esencialmente, por un sentido más crítico respecto a la formación y a los educadores, manifestado claramente en la entrevista. El perfil B, con los mismos profesores orientadores que A, está marcado por una trayectoria académica en el área de humanidades, pero manifestando un cierto agrado por las ciencias. La trayectoria académica de $\mathrm{D}$ es parecida a los casos B y C: D está marcado por la predilección por las ciencias, aumentada - probablemente- por la presencia del profesor cooperante, que ha mostrado su interés y su formación permanente. Designamos el perfil correspondiente a las prácticas de B y D como ciencia ilustrada. En el caso $\mathrm{C}$, cuyo perfil de prácticas pedagógicas designamos como ciencia descriptiva, se intuye la relación entre su trayectoria académica en el área de humanidades, su limitado entusiasmo por las ciencias y su escasa evolución durante la formación inicial.

Figura 1

Modelo de la investigación.

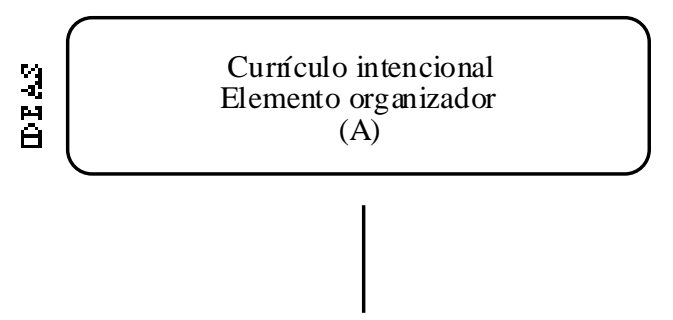

Análisis de contenido

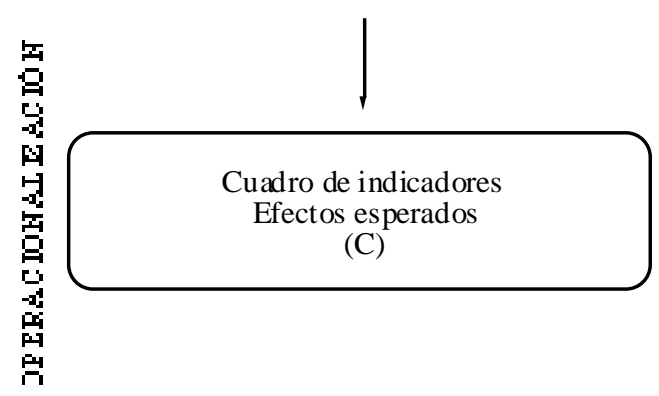

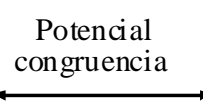

Análisis de congruencia

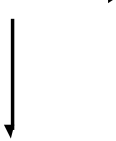

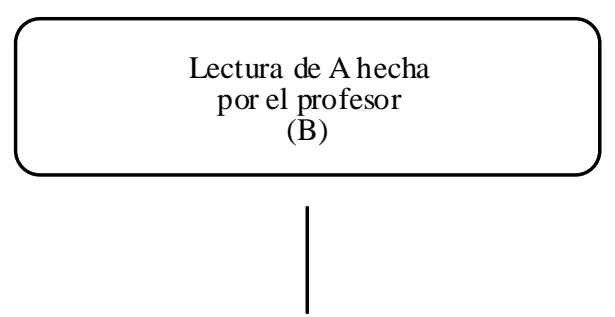

Observación - comportamiento

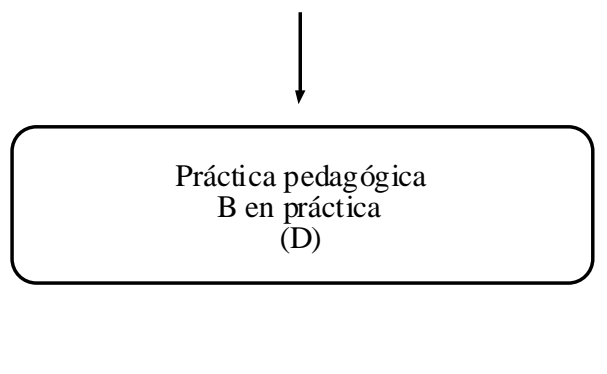

Nuevas ideas para la formación de profesores 


\section{CONCLUSIONES Y RECOMENDACIONES PARA LA FORMACION DE PROFESORES}

Del estudio realizado destaca que las prácticas pedagógicas (PP) realizadas por los profesores en período de formación (PF) están todavía muy lejos del cuadro teórico tomado como referencia, es decir, de acuerdo con la figura 1; el currículo intencional y el operacional están también bastante lejos de ser congruentes. Los tres perfiles identificados, que no pretenden anular necesariamente otras posibilidades, pueden servir como elementos de reflexión, teniendo en cuenta que ayudan a racionalizar propuestas de cambio en la formación del profesorado. Una vez entendido que para los educadores es importante comprender la relación entre la propuesta de innovación curricular, los resultados de la investigación y la formación anterior (Cachapuz, 1987), se vislumbra un largo camino por recorrer en relación con la formación de profesores de primer ciclo de enseñanza básica en el área de ciencias naturales. Es en este ámbito donde sugerimos algunas recomendaciones.

\section{Formación científica}

El primer punto que nos parece importante es la formación académica de base. Como bien ha demostrado Cachapuz (1992), esta formación es deficiente e inadecuada para las exigencias de los nuevos programas. Los resultados del presente estudio corroboran esta conclusión. Existe, pues, una necesidad de reestructuración de los currículos de formación científica inicial, adecuada a las necesidades de los programas y con una perspectiva crítica y actual. No se trata únicamente de añadir más horas, sino de posibilitar algo mucho más importante: realizar una gestión curricular diferente, favoreciendo una perspectiva de reflexión de los conocimientos absolutamente crítica.

\section{La enseñanza de las ciencias como trabajo científico}

Existe algo en común en las representaciones que los profesores en período de formación se hacen de la enseñanza de las ciencias, es decir, en todos aparece una visión empirista de la construcción del conocimiento. Todos ellos creen que los niños tienen necesidad de actividad, pero la identifican con la realización de experimentos y la observación de materiales ilustrativos. Tienen representaciones parecidas relativas a la imagen de buenas clases de ciencias, considerando la experimentación y la observación como los aspectos fundamentales y no dando importancia al papel de las hipótesis y de la previsión. Debido a la persistencia de estas perspectivas epistemológicas en los profesores, es necesario que se lleve a cabo un debate abierto, durante la etapa de formación, referente a la construcción del conocimiento y a la naturaleza del trabajo científico junto con sus implicaciones en la enseñanza-aprendizaje de las ciencias. Un debate de estas características debería plantearse mezclando los conocimientos teóricos con la epistemología de las prácticas, de manera que facilitara una recontextualización.

\section{Representación del profesor del primer ciclo de enseñanza básica}

Para todos los PF, en mayor o menor grado, aparece una representación del profesor de primer ciclo de enseñanza básica (CEB) que corresponde a un modelo tradicional, en el cual se da mucha importancia al respeto y al silencio impuesto de manera autoritaria y que concede prioridad al discurso informativo del profesor sobre la actividad de los alumnos. La práctica profesional surge como una situación especial que ni tan sólo puede ser tenida en cuenta por ser considerada una situación artificial. Todos coinciden en que los profesores de primer CEB actúan según este modelo de repetición e incluyen en este modelo a los profesores orientadores de la práctica profesional. Admiten que fatalmente, más pronto o más tarde, se adaptarán a este modelo de profesor, como si fuera inevitable, tal como muestra la práctica y la experiencia. ¡Parece ser que lo consideran eficaz!

Sería relevante que durante la formación los PF accedieran a diversas estrategias de enseñanza de las ciencias, para que no se limitaran al modelo del orientador. La defensa del pluralismo metodológico ha de pasar también por experiencias ricas y diversificadas que promuevan la reflexión sobre las estrategias de enseñanza de las ciencias. Estos aspectos tienen implicaciones, como veremos más adelante, en la formación de profesores.

\section{Representación de los nuevos programas}

Los profesores en formación no son conscientes de la importancia de los nuevos objetivos para la enseñanza de las ciencias propuestas en los nuevos programas. Desconocen los textos y parecen considerar que un programa es un conjunto de contenidos y objetivos preparado para ser consumido. Muestran una falta de preparación para poder explorar la versatilidad de los programas a través de los principios orientadores preconizados en los mismos. La discusión intencional sobre los nuevos programas ha de tener un espacio en los cursos de formación de profesores, para favorecer el contraste con la práctica profesional. Creemos que la formación de profesores, en esta década de los noventa, debería fundamentarse en una reflexión sobre los principios orientadores del aprendizaje, que ya se han explicitado en los textos de las reformas curriculares.

\section{Organización curricular de los cursos de formación de profesores}

Todos los profesores en formación consideran que han recibido una formación demasiado teórica y que han tenido pocas asignaturas del ámbito de ciencias. Están convencidos de que tienen una preparación más adecuada para la práctica profesional en otras áreas curriculares (por ejemplo, en lengua materna). En concreto, aribuyen la inadecuación del curso a la falta de asignaturas de metodología. No se da mucha importancia al curso inicial, por lo que se refiere a la contribución de las asignaturas del área científica, y puesto que no hubo una 
metodología específica para la enseñanza de las ciencias, parecen recordar mejor las orientaciones, recomendaciones y ejemplos utilizados por los orientadores.

Parece, pues, oportuno introducir una asignatura de metodología de la enseñanza de las ciencias en el curso de formación de profesores del primer ciclo de enseñanza básica, con el fin de disponer de tiempo para concretar, simular, observar, discutir, reformular, reflexionar, experimentar, autoevaluarse y avanzar en la integración teoría- práctica que necesariamente se llevará a cabo, directa y más intensamente, a final del curso (3r. año). Esto significa conceder a las ciencias un estatus de igualdad respecto a otras áreas curriculares -por ejemplo, la lengua materna y las matemáticas-así como unos objetivos propios.

\section{Formación de formadores}

Para que se produzca un cambio de rumbo en la formación de los profesores, otro aspecto a tener en cuenta sería proceder a la formación de formadores y a la evaluación de los efectos de esta formación. Es indispensable formar a los profesores colaboradores con relación al área de ciencias, para garantizar una coherencia en la formación, organizando cursos especializados, siempre con una perspectiva de integración de los componentes científico y didáctico.

El éxito de los cambios preconizados por la reforma curricular dependerá de la capacidad y disponibilidad de las instituciones de formación de profesores para incentivar la articulación entre investigación e innovación, haciéndolas atractivas a todos los profesores y, en particular, a los que formaron inicialmente, proporcionándoles así una formación continuada y permanente para renovar la práctica pedagógica.

\section{NOTA}

Este artículo ha sido traducido del portugués por Anna Costa.

\section{REFERENCIAS BIBLIOGRÁFICAS}

ABELL, S. y ROTH, M. (1992). Constraints to Teaching Elementary Science: A Case Study of a Science Enthusiast Student Teacher. Science Education, 76(6).

CACHAPUZ,A.F. (1987). Articulação, Investigação Educacional/ Práticas Educativas: Problemática e Perspectiva. Actas do Colóquio As Ciências da Educação e a Formação de professores, Dezembro de 1986. Lisboa: GEP-Ministério da Educação, pp. 68-79.

CACHAPUZ, A.F. (1992). Improving Primary Science Teaching in Portugal. Primary Science Review, 23, pp. 32-34.

CARRASCOSA, J. y GIL, D. (1985). La «metodologia de la superficialitat» i l'aprenentatge de les ciències. Enseñanza de las Ciencias, 3, pp. 113-120.

CLEMINSON, A. (1990). Establishing an Epistemological Base for Science Teaching in the light of Contemporary Notions of the nature of Science and of how children learn Science. Journal of Research in Science Theaching. 27(5), pp. 429-445.

COSTA, N., VEIGA, L., MARQUÉS, L., CRUZ, N., CABRITA, A. y CACHAPUZ, A. (1993). Formação inicial em Ciencias de Professores do $1^{\circ}$ Ciclo do Ensino Básico. Monografia no ambito do Projecto CInEB. Aveiro: Universidade de Aveiro.
CRONIN-JONES, L. y SHAW, JR. E. (1992). The influence of Methods Instruction on the beliefs of preservice Elementary and Secondary Science Teacher. Preliminary Comparative Analysis. Studies in Science and Mathemanics, 92(1).

DUSCHL, R. (1983). The elementary level science methods course: Breeding ground of an apprehension toward science? Journal of Science Teaching, 20, pp. 745-754.

FOUREZ, G. (1988). La Construction des Sciences. Bruxelles: De Boeck-Wesmael.

GEP EDUCAÇÃO (1989). Prática Pedagógica: Análise da Situação, Série B: Dinâmica do Sistema Educativo, 13 Volume, $2^{\circ}$ Volume. Lisboa: Ministério da Educacão.

GEYMONAT, L. (s.d.) Elementos de Filosofia da Ciência. Lisboa: Gradiva.

HARLEN, W. (1985). An International Workshop on Primary Science. Actas: Science and Technology Education and future Human needs. Indi: Ed. Harlen.

HEWSON, P. W. y TORLEY, N.R. (1989). The conditions of Conceptual Change in the Classroom. International Journal of Science Education, 11, Special Issue, pp. 541-553. 
IIE, 1992. Avaliação da Reforma - A opinião dos Professores, $1^{\circ}$ Ciclo. Lisboa: IIE.

JORGE, M.M.P. (1987). Eficácia do Modelo Integrado na Formação de Professores de Ciências da Natureza. Tesis de maestría (sin publicar). Universidade do Minho.

LBSE. Lei n ${ }^{\circ}$ 46/86 de 14 de Outubro. Diário da República, $1^{\text {a }}$ Série, núm. 237.

NOVAK, J.D. (1977). A Theory of Education. Ithaca: Cornell University Press.

PAIXÃO, M F. (1993). Os Desafios da Reforma Curricular e a Formacão de Professores de Ciencias da Natureza do $1^{\circ}$ Ciclodo Ensino Básico. A Prática Pedagógica como indicador de mudanças necessárias. Tesis de maestría (sin publicar). Universidade de Aveiro.

PÉREZ, D.G. (1986). La metodología cientifica y la enseñanza de las ciencias, unas relaciones controvertidas. Enseñanza de las Ciencias, 4 (2), pp. 111-121.
SANTOS, B.S. (1987). Um discurso sobre as Ciencias. Porto: Edicões Afrontamento.

STEFANICH, G. y KELSEY, K. (1989). Improving Science Attitudes of Preservice Elementary Teachers. Science Education, 73(2).

TAYLOR, R. y CHEUNG, K.C. (1991). Towards a Humanistic Constructivist model of Science learning: Changing perspectives and Research Implications. Journal of Curriculum Studies, 23(1), pp. 721-740.

UNESCO (1986). The place of Science and Technology in School Curricula: A global Survey. París: UNESCO.

WALLACE, J. y LOUDEN, W. (1992). Science Teaching and Teachers' Knowledge: Prospects for Reform of Elementary Classrooms. Science Education, 76(5), pp. 507-522.

WH1 TE, R. (1988). Learning Science. Oxford: Basil Blackwell.

YIN, R. (1987). Case Study Research: Design and Methods. Londres: Sage Publications.

[Artículo recibido en mayo de 1994 y aceptado en septiembre de 1998.] 